\section{Small Angle Scattering of Electrons in Helium}

Is July $1933^{1}$ one of us (R. W.), in collaboration with T. Emmerson and J. E. Taylor, pointed out that, in agreement with Mr. S. N. Van Voorhis ${ }^{2}$, we had been obtaining curious scattering effects using narrow electron beams passing through helium at low pressures.

The effect, which was very marked at energies of between 100 and 200 electron volts, consisted in a very obvious maximum in the number of inelastically scattered electrons at a few degrees out from the main beam. This result was so remarkable and unexpected as to demand further and much closer examination, and with that end in view a special apparatus has been set up in this laboratory.

Briefly, the electron gun is rotated slowly and continuously by a synchronous electric motor, the scattered electrons, afterpassing through a slit system, being analysed by a magnetic field and the resulting spectrum recorded on a photographic film kept in continuous motion by a second synchronous motor. The result is a continuous record of all the scattered electrons both elastic and inelastic over the desired angular range; energy and number being simultaneously recorded in terms of position on the film and photographic density respectively.

The photograph here reproduced as Fig. 1 indicates the elegant kind of record which can be obtained by this method. The two separate spectra show

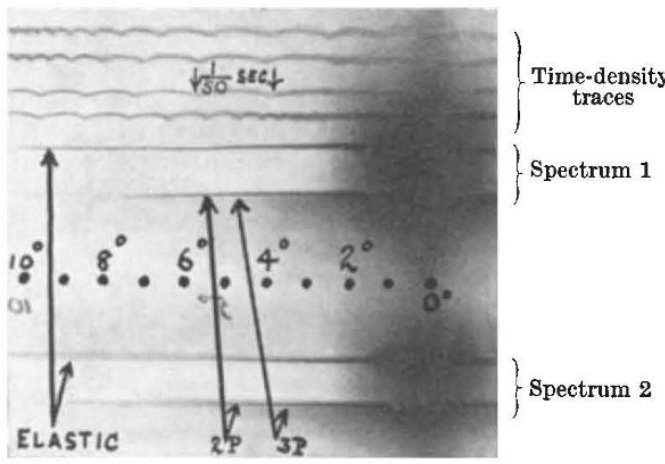

FIG. 1 .

the variation in probability up to $10^{\circ}$ from the main beam of elastic impacts, and those which have resulted in the two most likely transitions $(2 P$ and $3 P$ ). At the top of the record are to be seen four wavy traces which have been produced by sweeping the electrons (at zero beam setting) rapidly across the film with a time-marking device. By suitably accelerating the motion, a range of wave-lengths can be obtained, as shown, from which density-intensity information can be derived for interpreting the spectral traces themselves.

The anomalous results previously reported have not, so far, been confirmed with this more refined apparatus.

We have measured the relative probabilities of the elastic and inelastic $(2 P)$ collisions at angles up to

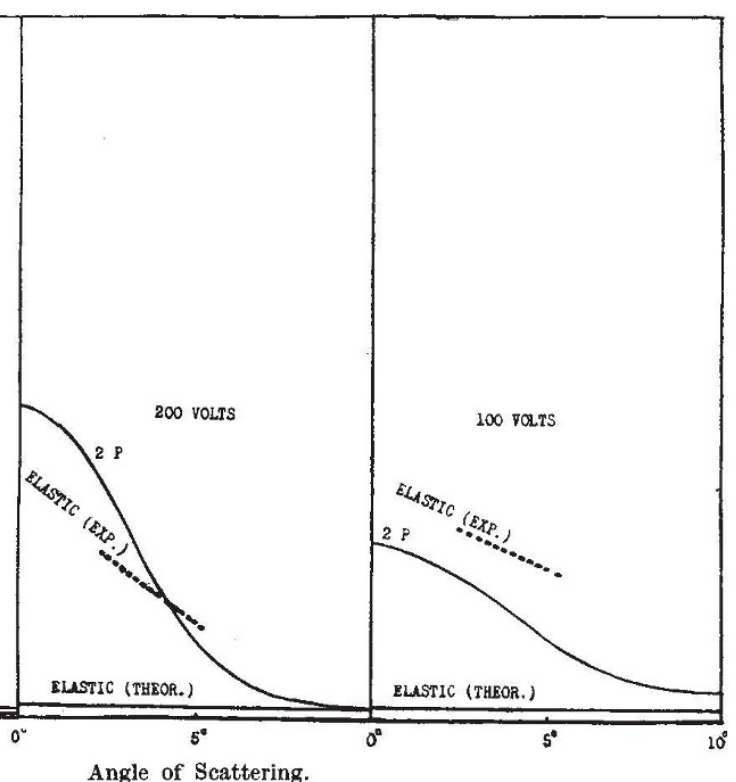

FIG. 2.

$10^{\circ}$ at energies of impact varying between 100 and 400 electron volts with the results shown in Fig. 2. Here, the inelastic probabilities given by the most recent form of theory (Massey and Mohr) ${ }^{3}$ have been taken as the basis and in agreement with our experimental results (full curves marked $2 P$ ). It will be seen how very different are the calculated (full lines) and experimental (dotted lines) values for the elastic probabilities. In particular, at the lower impacting energies this divergence is specially remarkable.

Physical Laboratories,

F. C. Poultney.

R. WhiddingtoN.

University, Leeds.

I NATURE, 132, 65, July 8, 1933.

3 Proc. Roy. Soc., A, 140, 613; 1933.

\section{Galvanometer Amplification by Photo-Cell}

In $1931^{1}$ I described the use of a differential photoelectric cell connected to a 'secondary' galvanometer for amplifying the movements of a 'primary' one. The only suitable cell available then was a cuprous oxide one, and the current produced by it was not great enough to allow a quick (and therefore insensitive) secondary galvanometer to be used; consequently, although sufficient amplification was obtained, the movements were slowed. This slowing of movements is an even greater objection to the thermal relay of Moll and Burger since the thermocouple introduces a further lag which the photo-cell does not.

I have recently tested a Weston 'photronic' cell for 